\title{
The heterotic string at high temperature (or with strong supersymmetry breaking)
}

\author{
Waldemar Schulgin ${ }^{a}$ and Jan Troost $^{b}$ \\ ${ }^{a}$ George and Cynthia Mitchell Institute for Fundamental Physics and Astronomy, \\ Texas A \& $M$ University, \\ College Station, TX 7r843-4242, U.S.A. \\ ${ }^{b}$ Laboratoire de Physique Théorique ${ }^{1}$ Ecole Normale Supérieure \\ 24 rue Lhomond, 75005 Paris, France \\ E-mail: schulgin@physics.tamu.edu, troost@lpt.ens.fr
}

AbStRaCt: Perturbative heterotic string theory develops a single complex tachyonic mode beyond the Hagedorn temperature. We calculate the quartic effective potential for this tachyonic mode at the critical temperature. Equivalently, we determine the quartic effective potential for strong supersymmetric breaking via anti-perdiodic boundary conditions for fermions on a small circle. We give many details of the heterotic tachyon scattering amplitudes, including a unitarity check to fix all normalization constants. We discuss difficulties in obtaining an effective action valid at all radii. We argue that in certain variables, the quartic term in the potential is radius independent. Speculations on the properties of a new strongly curved phase that could occur after tachyon condensation are offered.

Keywords: Superstrings and Heterotic Strings, Tachyon Condensation

ARXIV EPRINT: 1107.5316

\footnotetext{
${ }^{1}$ Unité Mixte du CNRS et de l'Ecole Normale Supérieure associée à l'université Pierre et Marie Curie 6, UMR 8549.
} 


\section{Contents}

1 Introduction $\quad 2$

2 The spectrum 2

2.1 Heterotic string theory at finite temperature 2

2.2 The partition function 3

2.2.1 The supersymmetric ten-dimensional partition function 3

2.2.2 Compactification on a circle 3

2.2.3 Twisting the periodicity conditions 3

2.3 The spectrum hands-on 5

$\begin{array}{lll}2.4 & \text { Summary of the low-energy excitations } & 7\end{array}$

$\begin{array}{lll}2.4 .1 & \text { At generic radius } & 7\end{array}$

$\begin{array}{lll}2.4 .2 & \text { At the self-dual radius } & 7\end{array}$

$\begin{array}{lll}2.4 .3 \text { Remark } & 7\end{array}$

$\begin{array}{ll}2.5 & \text { A note on spontaneous supersymmetry breaking }\end{array}$

3 Scattering amplitudes $\quad \mathbf{8}$

3.1 Vertex-operators 8

3.2 Amplitudes 9

3.2.1 Tachyon-tachyon-gravitational three-point amplitude $\quad 9$

$\begin{array}{ll}3.2 .2 \text { Tachyon four-point amplitude } & 10\end{array}$

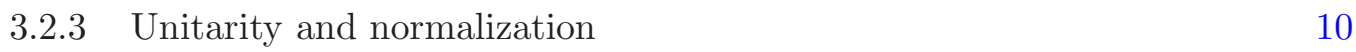

3.2.4 Tachyon-Tachyon-Gravitational-Gravitational four-point amplitude 11

$\begin{array}{lll}3.2 .5 & \text { Summary } & 12\end{array}$

4 The effective action $\quad 12$

$\begin{array}{lll}4.1 \text { The action } & 12\end{array}$

4.2 Expansion of the action around the flat background 13

$\begin{array}{lll}4.3 & \text { Cubic couplings } & 14\end{array}$

$\begin{array}{lll}4.4 \text { Remark } & 15\end{array}$

$\begin{array}{ll}\text { 4.5 A quartic coupling } & 15\end{array}$

4.5.1 Massless exchange contributions to the four-point tachyon amplitude 16

$\begin{array}{ll}\text { 4.5.2 The quartic term in the tachyon potential } & 17\end{array}$

$\begin{array}{lll}4.5 .3 & \text { Remark } & 17\end{array}$

$\begin{array}{lll}5 & \text { The effective action at smaller radii } & 18\end{array}$

$\begin{array}{ll}\text { 5.1 The effective action at the self-dual radius } & 18\end{array}$

$\begin{array}{ll}5.2 \text { A universal behaviour } & 18\end{array}$

$\begin{array}{lll}5.3 & \text { Field theory exchange diagrams } & 19\end{array}$

$\begin{array}{lll}5.3 .1 & \text { Universality } & 20\end{array}$

6 Conclusions and speculations $\quad 20$ 


\section{Introduction}

The high temperature phase of string theory is ill understood [1-3]. Beyond a critical temperature, the canonical ensemble is no longer well-defined. There is no known effective theory that describes the physics of strings at temperatures above the Hagedorn temperature. The effective degrees of freedom are not known.

While the problem is universal to all perturbative string theories, we wish to concentrate on the heterotic superstring. Due to the off-set in the left and right level-matching conditions, the momentum and winding of the heterotic string at finite temperature need to be non-zero at zero oscillator excitation. Therefore, the potentially tachyonic mode will become massive at both small and large radius. In fact, in the critical window only a single perturbatively tachyonic mode can develop. That is a property unique to the heterotic string. Moreover, the heterotic string has an enhanced gauge symmetry at self-dual radius.

The heterotic string at finite temperature has been studied before [1, 4-7]. It was argued on general grounds to exhibit a first order phase transition. Later on, non-perturbative potentials were proposed to describe the lower-dimensional heterotic string at finite temperature in a spontaneous supersymmetry breaking framework [8] and it was shown that one can sidestep the phase transition by introducing background potentials $[9,10]$.

In this paper, we will further analyze properties of the perturbative heterotic string at finite temperature and in equilibrium. Let us immediately remark that this is formally equivalent to thinking of the heterotic string as being compactified on a circle with periodic boundary conditions for the bosonic field theory degrees of freedom, and anti-periodic boundary conditions for the fermionic ones. We will find the second picture more convenient for the bulk of the paper, since it permits us to directly interpret all of the physical excitations. Thus, we will think of heterotic string at finite temperature as a superstring theory in which we broke supersymmetry through periodicity conditions (à la ScherkSchwarz).

We will calculate the quartic term in the tachyon effective potential at the critical radius. To that end, we plough through some of the detailed calculations, fix all normalizations, perform a unitarity check, et cetera. We give the details here since we were not able to extract them easily from the literature. We then use the experience gained to offer speculative comments on what might happen beyond the Hagedorn radius.

\section{The spectrum}

In this section, we rederive the partition function of the theory under study, and its lowenergy modes in the regimes of interest to us.

\subsection{Heterotic string theory at finite temperature}

The partition function for a system at finite temperature and in equilibrium is given by the path integral on space times a circle, with periodic boundary conditions for the bosonic field theoretic degrees of freedom and anti-periodic boundary conditions for the fermionic ones. The inverse radius of compactification is $(2 \pi$ times) the temperature (at least at 
temperatures low compared to the string scale). Equivalently, we can think rather of a theory at zero temperature, compactified on a circle, with twisted periodicity conditions that will break supersymmetry. The radius of compactification sets the supersymmetry breaking scale. When discussing the problem at hand, we will mostly take the latter point of view, such that we can truely pick a light-cone gauge, and talk about all excitations as physical excitations of our system.

We determine the spectrum of the theory at generic radius of compactification $R$. We do this on the one hand by analyzing the exact partition function, and on the other hand by a hands-on analysis of the physical Hilbert space.

\subsection{The partition function}

\subsubsection{The supersymmetric ten-dimensional partition function}

We have that the toroidal partition function for heterotic string theory on $\mathbb{R}^{9,1}$ is:

$$
Z_{T^{2}}=i V_{10} \int \frac{d \tau d \bar{\tau}}{4 \tau_{2}} \frac{1}{\left(4 \pi^{2} \alpha^{\prime} \tau_{2}\right)^{5}} \frac{1}{|\eta|^{16}} \frac{\Gamma_{16}}{\eta^{16}} \frac{1}{2} \sum_{a, b=0}^{1}(-1)^{a+b+a b} \frac{\bar{\vartheta}\left[\begin{array}{l}
a / 2 \\
b / 2
\end{array}\right]^{4}}{\bar{\eta}^{4}} .
$$

There is a prefactor corresponding to the integration over 10 zero-modes, and there are 8 sets of bosonic transverse oscillator excitations. The last factor corresponds to the GSO projected right-moving worldsheet fermions. The factor $(-1)^{b}$ corresponds to the twist by the right-moving worldsheet fermion number while the factor $(-1)^{a}$ is added to represent the minus sign due to a space-time fermion loop. The left-movers of the heterotic superstring moreover exhibit a sixteen-dimensional charge lattice $\Gamma_{16}$ for compact bosons corresponding either to the $E_{8} \times E_{8}$ lattice or to the $\operatorname{Spin}(32) / \mathbb{Z}_{2}$ lattice.

\subsubsection{Compactification on a circle}

These formulas represent the partition function containing all zero modes and all oscillator excitations transverse to the light-cone. Compactification to a spatial circle with radius $R$ and periodic fields leads to the partition function:

$$
Z_{T^{2}}=2 i \pi R V_{9} \int \frac{d \tau d \bar{\tau}}{4 \tau_{2}} \frac{1}{\left(4 \pi^{2} \alpha^{\prime} \tau_{2}\right)^{5}} \frac{1}{|\eta|^{16}} \frac{\Gamma_{16}}{\eta^{16}} \frac{1}{2} \sum_{a, b=0}^{1}(-1)^{a+b+a b} \frac{\bar{\vartheta}\left[\begin{array}{l}
a / 2 \\
b / 2
\end{array}\right]^{4}}{\bar{\eta}^{4}} \sum_{m, w=-\infty}^{+\infty} e^{-\frac{\pi R^{2}|m-w \tau|^{2}}{\alpha^{\prime} \tau_{2}}} .
$$

The quantum number $w$ corresponds to the winding and the quantum number $m$ is Poisson dual to the momentum. The argument of the exponential represents the classical action of the worldsheet that winds around the two cycles of the torus with modular parameter $\tau$.

\subsubsection{Twisting the periodicity conditions}

We now wish to change the periodicity conditions on the fields. The field theory bosons remain periodic while the field theory fermions are taken to be anti-periodic on the circle. This breaks supersymmetry at the length scale $R$. It is equivalent to considering the 
partition sum of the system at equilibrium and at finite temperature. In the process, we want to retain modular invariance of the integrand of the one loop vacuum amplitude. These requirements lead to an extra phase factor depending on the sector of the theory and the winding numbers of the string [1]:

$$
U_{a b}(m, w)=\frac{1}{2}(-1)^{a b}\left(1+(-1)^{a+w}+(-1)^{b+m}-(-1)^{a+b+m+w}\right) .
$$

It gives rise to the partition function $[1,4,5]$ :

$$
\begin{aligned}
Z_{T^{2}}= & 2 i \pi R V_{9} \int \frac{d \tau d \bar{\tau}}{4 \tau_{2}} \frac{1}{\left(4 \pi^{2} \alpha^{\prime} \tau_{2}\right)^{5}} \frac{1}{|\eta|^{16}} \frac{\Gamma_{16}}{\eta^{16}} \sum_{m, w=-\infty}^{+\infty} \sum_{a, b=0}^{1} \\
& \times \frac{1}{4}(-1)^{a+b}\left(1+(-1)^{a+w}+(-1)^{b+m}-(-1)^{a+b+m+w}\right) \frac{\bar{\vartheta}\left[\begin{array}{c}
a / 2 \\
b / 2
\end{array}\right]}{\bar{\eta}^{4}} e^{-\frac{\pi R^{2}|m-w \tau|^{2}}{\alpha^{\prime} \tau_{2}}} .
\end{aligned}
$$

One can easily check that for zero winding number $w=0$ (and for any even $w$ ), there is no change due to the extra phase factor for the bosons $(a=0)$. For the fermions, one obtains an extra factor of $(-1)^{m}$ in this sector, which corresponds to anti-periodic fermions winding $m$ times. This agrees with the requirements in the point particle limit. The remaining phases are fixed by modular invariance. For bosons with odd winding $w$, we find that the GSO projection is reversed (due to an extra phase $(-1)^{b}$ ) and that an extra phase $(-1)^{m}$ is added to the partition sum. After Poisson resummation on the summation variable $m$, we find the partition sum in Hamiltonian form:

$$
\begin{aligned}
Z_{T^{2}}= & i V_{9} \int \frac{d \tau d \bar{\tau}}{16 \tau_{2}} \frac{1}{\left(4 \pi^{2} \alpha^{\prime} \tau_{2}\right)^{9 / 2}} \frac{\Gamma_{16}}{\eta^{24} \bar{\eta}^{12}} \\
& \times\left(\sum_{n, w=-\infty}^{\infty}\left(\left(1+(-1)^{w}\right) q^{\alpha^{\prime} p_{L}^{2} / 4} \bar{q}^{\alpha^{\prime} p_{R}^{2} / 4}+\left(1-(-1)^{w}\right) q^{\alpha^{\prime} \tilde{p}_{L}^{2} / 4} \bar{q}^{\alpha^{\prime} \tilde{p}_{R}^{2} / 4}\right) \bar{\vartheta}_{3}^{4}\right. \\
& \quad-\sum_{n, w=-\infty}^{\infty}\left(\left(1+(-1)^{w}\right) q^{\alpha^{\prime} p_{L}^{2} / 4} \bar{q}^{\alpha^{\prime} p_{R}^{2} / 4}+\left(-1+(-1)^{w}\right) q^{\alpha^{\prime} \tilde{p}_{L}^{2} / 4} \bar{q}^{\alpha^{\prime} \tilde{p}_{R}^{2} / 4}\right) \bar{\vartheta}_{4}^{4} \\
& \left.-\sum_{n, w=-\infty}^{\infty}\left(\left(1-(-1)^{w}\right) q^{\alpha^{\prime} p_{L}^{2} / 4} \bar{q}^{\alpha^{\prime} p_{R}^{2} / 4}+\left(1+(-1)^{w}\right) q^{\alpha^{\prime} \tilde{p}_{L}^{2} / 4} \bar{q}^{\alpha^{\prime} \tilde{p}_{R}^{2} / 4}\right) \bar{\vartheta}_{2}^{4}\right)
\end{aligned}
$$

where the momenta are defined by:

$$
p_{L, R}=\frac{n}{R} \pm \frac{w R}{\alpha^{\prime}}, \quad \tilde{p}_{L, R}=\frac{n-\frac{1}{2}}{R} \pm \frac{w R}{\alpha^{\prime}} .
$$

We see that both the GSO projection and the momentum lattice in the right-moving NS sector are standard at even winding number. When the winding is odd, however, we have that the GSO projection is reversed in the right-moving NS sector, and that the momenta are shifted by half. 


\subsection{The spectrum hands-on}

The mass shell conditions for the left-movers and the NS and R sector right-movers are:

$$
\begin{aligned}
& m^{2}=-\frac{4}{\alpha^{\prime}}+\frac{4 N^{\text {left }}}{\alpha^{\prime}}+\left(\frac{n}{R}+\frac{w R}{\alpha^{\prime}}\right)^{2}, \\
& m^{2}=-\frac{2}{\alpha^{\prime}}+\frac{4 N_{\mathrm{NS}}^{\text {right }}}{\alpha^{\prime}}+\left(\frac{n}{R}-\frac{w R}{\alpha^{\prime}}\right)^{2}, \\
& m^{2}=\frac{4 N_{\mathrm{R}}^{\text {right }}}{\alpha^{\prime}}+\left(\frac{n}{R}-\frac{w R}{\alpha^{\prime}}\right)^{2} .
\end{aligned}
$$

The sectors refer to the sectors for the worldsheet fermion superpartners of the ten spacetime coordinates. We will concentrate on the space-time bosons. These necessarily arise from the right-moving NS sector. Thus we can concentrate on the left-movers, and the NS sector right-movers. For these excitations, we necessarily have:

$$
N_{N S}^{\text {right }}-N^{\text {left }}+\frac{1}{2}-n w=0
$$

For concreteness, we will concentrate on the $\operatorname{Spin}(32) / \mathbb{Z}_{2}$ heterotic superstring in the following. We introduce bosonic fields $X^{A}$ with oscillators $\alpha^{A}$, right-moving worldsheet fermions $\psi^{A}$, and 32 left-moving worldsheet fermions $\lambda^{a}$. The latter can be periodic or anti-periodic and are taken to satisfy $e^{i \pi F_{\lambda}}=1$, in order to give rise to a partition function factor that is modular invariant by itself. Note that the periodic fermions $\lambda$ do not give rise to lowlying excitations. We will split the space-time index $A$ into the index $\mu$ running over the non-compact directions $0,1 \ldots, 8$ and the index 9 , in the compactified direction.

In the left-moving sector, we have the low-lying modes:

\begin{tabular}{|cc|l|}
\hline$N^{\text {left }}$ & $m^{2}$ & states \\
\hline 0 & $-\frac{4}{\alpha^{\prime}}+\left(\frac{n}{R}+\frac{w R}{\alpha^{\prime}}\right)^{2}$ & $|n, w\rangle$ \\
1 & $\left(\frac{n}{R}+\frac{w R}{\alpha^{\prime}}\right)^{2}$ & $\left(\alpha_{-1}^{\mu, 9} \quad\right.$ or $\left.\quad \lambda_{-1 / 2}^{a} \lambda_{-1 / 2}^{b}\right)|n, w\rangle$. \\
\hline
\end{tabular}

In the right-moving NS sector we find:

\begin{tabular}{|cc|l|}
\hline$N_{N S}^{\text {right }}$ & $m^{2}$ & states \\
\hline 0 & $-\frac{2}{\alpha^{\prime}}+\left(\frac{n}{R}-\frac{w R}{\alpha^{\prime}}\right)^{2}$ & $|n, w\rangle$ \\
$\frac{1}{2}$ & $\left(\frac{n}{R}-\frac{w R}{\alpha^{\prime}}\right)^{2}$ & $\psi_{-1 / 2}^{\mu, 9}|n, w\rangle$. \\
\hline
\end{tabular}

We tensor the left-movers with the right-moving NS sector to obtain level-matched states in the spectrum, satisfying the modified GSO projection. We will look for states that can be massless or tachyonic at a given radius $R$. We start out with the left and right ground state. To level match, we need $n w=1 / 2$. That gives two states labelled by (shifted) 
momenta $(n, w)= \pm(1 / 2,1)$. They satisfy the (reversed) GSO projection. They represent a complex scalar $T^{ \pm}$of mass squared:

$$
m^{2}=\frac{1}{4 R^{2}}+\frac{R^{2}}{\alpha^{\prime 2}}-\frac{3}{\alpha^{\prime}} .
$$

The complex scalar is massless at the Hagedorn radii:

$$
\frac{R_{H}}{\sqrt{\alpha^{\prime}}}=1 \pm \frac{1}{\sqrt{2}} .
$$

At a radius between these two values, the complex scalar is tachyonic, and we will loosely refer to this scalar as the tachyon. When we attempt to combine the right-moving ground state with the left-moving first excited state (at level one half), we cannot satisfy GSO and the conditions on the fermionic excitations $\lambda$. The same is true if we reverse left and right. If we take both to be at the first excited level, there will again be no solution satisfying all consistency conditions.

The second set of states arises from the right-moving ground states, and the left-moving state at level 1 . We need $n w=-1 / 2$. There are two solutions, namely $(n, w)= \pm(1 / 2,-1)$. The mass squared of the states is:

$$
m^{2}=\frac{\left(-2 R^{2}+\alpha\right)^{2}}{4 R^{2} \alpha^{\prime 2}}
$$

The states becomes massless at the self-dual radius:

$$
\frac{R_{\mathrm{sd}}}{\sqrt{\alpha^{\prime}}}=\frac{1}{\sqrt{2}} \text {. }
$$

At this radius they have left-moving momentum equal to zero and right-moving momentum $\tilde{p}_{R}=\sqrt{2 / \alpha^{\prime}}$. The states represent two vectors, a complex scalar and a complex scalar in the adjoint of $\operatorname{Spin}(32) / \mathbb{Z}_{2}$ :

$$
\left(\alpha_{-1}^{\mu} \quad \text { or } \quad \alpha_{-1}^{9} \quad \text { or } \quad \lambda_{-1 / 2}^{a} \lambda_{-1 / 2}^{b}\right) \otimes\left| \pm \frac{1}{2}, \mp 1\right\rangle .
$$

The last possibility is to have the level $1 / 2$ state on the right, and the level 1 state on the left. We have then the constraint $n w=0$, and we can concentrate on the solution $(n, w)=0$. This solution corresponds to the states:

$$
\left(\alpha_{-1}^{\mu} \quad \text { or } \quad \alpha_{-1}^{9} \quad \text { or } \quad \lambda_{-1 / 2}^{a} \lambda_{-1 / 2}^{b}\right) \otimes\left(\psi_{-1 / 2}^{\nu} \quad \text { or } \quad \psi_{-1 / 2}^{9}\right)|0,0\rangle
$$

which are the 9-dimensional graviton, dilaton and anti-symmetric two-tensor, as well as two U(1) Kaluza-Klein vectors, a scalar radion, an scalar adjoint of $\operatorname{Spin}(32) / \mathbb{Z}_{2}$ as well as a vector in the adjoint of $\operatorname{Spin}(32) / \mathbb{Z}_{2}$. There can be no second tachyonic mode at any radius [11]. Going higher in oscillation number will automatically raise the mass squared to a positive number.

It is straightforward to check that our hands-on description agrees with the first terms in the expansion of the partition function in equation (2.4). 


\subsection{Summary of the low-energy excitations}

We summarize the low-energy excitations that we will be interested in.

\subsubsection{At generic radius}

We broke supersymmetry in the $\operatorname{Spin}(32) / \mathbb{Z}_{2}$ heterotic superstring via compactification on a circle of radius $R$ with twisted periodicity conditions. The nine-dimensional field content of the theory includes the nine-dimensional metric $g_{\mu \nu}$, the anti-symmetric tensor field $b_{\mu \nu}$, the Kaluza-Klein gauge fields $A_{\mu}^{g, b}$ as well as the ten-dimensional dilaton $\Phi$ and a radion field $e^{2 \sigma}=g_{99}$. We also have the $\operatorname{Spin}(32) / \mathbb{Z}_{2}$ adjoint gauge field and adjoint scalar.

We also have a complex tachyon $T^{ \pm}$whose mass depends on the radius of compactification. At the Hagedorn radii, it becomes massless. In the interval between Hagedorn radii, the tachyon has negative mass squared.

\subsubsection{At the self-dual radius}

At the self-dual radius, we find two extra massless vector fields, two extra massless scalars as well as two extra massless $\operatorname{Spin}(32) / \mathbb{Z}_{2}$ adjoint scalars. The gauge group at the radius $R_{\mathrm{sd}}=\sqrt{\alpha^{\prime} / 2}$ enhances to $\mathrm{SU}(2)_{R} \times \mathrm{U}(1)_{L} \times \mathrm{Spin}(32) / \mathbb{Z}_{2}$. At this radius, the tachyon leftmoving momentum is $\sqrt{2 / \alpha^{\prime}}$ while the right-moving momentum is zero. The tachyon is a $\mathrm{U}(1)_{L}$ charged field and a $\mathrm{SU}(2)_{R}$ singlet. The two extra massless scalars that we obtain at this radius fill out a $(3,0,1)$ multiplet $^{1}$ of the group $\mathrm{SU}(2)_{R} \times \mathrm{U}(1)_{L} \times \mathrm{Spin}(32) / \mathbb{Z}_{2}$. Indeed, these states have the same transformation properties as the vectors that fill out an adjoint of $\mathrm{SU}(2)_{R}$. The $\operatorname{Spin}(32) / \mathbb{Z}_{2}$ adjoint scalars combine into a $(3,0$, Adj $)$ multiplet.

\subsubsection{Remark}

One of our motivations to study the Hagedorn problem in the heterotic superstring is the fact that at most one tachyon occurs at generic radius [11].

\subsection{A note on spontaneous supersymmetry breaking}

While the Scherk-Schwarz reduction that we performed can be described as a theory in which supersymmetry is broken spontaneously, a word of caution may be needed. Indeed, the theory is supersymmetric at infinite radius (which we can think of as the vacuum expectation value of a scalar field) and therefore supersymmetry is broken spontaneously in the theory, by giving a finite vacuum expectation value to the radius field. But we must note as well that certain fields are infinitely massive in the supersymmetric theory. Those fields include all winding modes, and in particular the tachyon.

From the perspective of the original ten dimensional theory these modes are nonlocal. These degrees of freedom are hard to describe in terms of a local supersymmetric Lagrangian with supersymmetry spontaneously broken. This phenomenon is typical of string theory (in contrast to field theory).

\footnotetext{
${ }^{1}$ We denote the dimension of the group, except for $\mathrm{U}(1)_{L}$, where we indicate the charge.
} 


\section{Scattering amplitudes}

Our next goal will be to understand aspects of the effective action for the low-energy excitations of the theory. Interesting phenomena are bound to happen near the Hagedorn radii, where the tachyon becomes massless, and at the point of enhanced symmetry. We want to reconstruct the effective action for the low-energy excitations, and in particular for the tachyons. When we concentrate on the Hagedorn radius $R_{H} / \sqrt{\alpha^{\prime}}=1+1 / \sqrt{2}$, the tachyons are massless. The tachyons are charged under the gauge fields arising from the reduction of the metric and the two-form. They are moreover coupled to the graviton, the dilaton and the radion. We will compute the three-point amplitudes between the tachyons and the other massless fields, to fix all normalizations in the effective action at the Hagedorn point. We then also calculate the four point tachyon amplitude to determine the coefficient of the quartic term in the effective tachyon potential. Additionally, we will fix the overall normalization of the amplitudes through a unitarity check. We perform the scattering amplitude calculations for a generic radius, and restrict to the Hagedorn point when the time has come.

\section{$3.1 \quad$ Vertex-operators}

To compute the scattering amplitudes of tachyons and gravitational modes, we need their vertex operators. The states and the vertex operators for the tachyons in the minus one picture are:

$$
\begin{array}{r}
\left|0 ; k ; n=\frac{1}{2}, w=1\right\rangle_{-1}: \quad V_{T+}^{-1}=\frac{1}{\sqrt{2 \pi R}} e^{i k_{\mu} X^{\mu}(z, \bar{z})} e^{i k_{9, L} X^{9}(z)+i k_{9, R} X^{9}(\bar{z})} e^{-\tilde{\phi}(\bar{z})}, \\
\left|0 ; k ; n=-\frac{1}{2}, w=-1\right\rangle_{-1} \quad: \quad V_{T-}^{-1}=\frac{1}{\sqrt{2 \pi R}} e^{i k_{\mu} X^{\mu}(z, \bar{z})} e^{-i k_{9, L} X^{9}(z)-i k_{9, R} X^{9}(\bar{z})} e^{-\tilde{\phi}(\bar{z})} .
\end{array}
$$

The state-operator map maps normalized states on the left to normalized vertex operators on the right. On the right, we have added the extra factor $\frac{1}{\sqrt{2 \pi R}}$ because in normalizing the vertex operator, we integrate over the compact direction. In a scattering amplitude, one power of the ten-dimensional string coupling constant accompanies every vertex operator. For the tachyon, we will take it to be $g_{T}^{10}=\sqrt{2 \pi R} g_{T}$. For the gravitational sector we will use $g_{c}^{10}=\sqrt{2 \pi R} g_{c}$. Unitarity requires that the equality $g_{c}=g_{T}$ holds. We will perform this unitarity check.

The vertex operators for the metric, the dilaton, the anti-symmetric tensor, the KaluzaKlein gauge bosons and the radion in the minus one picture are:

$$
\begin{aligned}
\left|N=1, \tilde{N}=\frac{1}{2} ; k ; n=0, w=0\right\rangle_{-1} & : i\left(\frac{2}{\alpha^{\prime}}\right)^{1 / 2} \frac{1}{\sqrt{2 \pi R}} e_{\mu \nu} \partial X_{L}^{\mu}(z) \tilde{\psi}^{\nu}(\bar{z}) e^{i k_{\rho} X^{\rho}(z, \bar{z})} e^{-\tilde{\phi}(\bar{z})} \\
& i\left(\frac{2}{\alpha^{\prime}}\right)^{1 / 2} \frac{1}{\sqrt{2 \pi R}} e_{\mu 9} \partial X_{L}^{\mu}(z) \tilde{\psi}^{9}(\bar{z}) e^{i k_{\rho} X^{\rho}(z, \bar{z})} e^{-\tilde{\phi}(\bar{z})} \\
& i\left(\frac{2}{\alpha^{\prime}}\right)^{1 / 2} \frac{1}{\sqrt{2 \pi R}} e_{9 \mu} \partial X_{L}^{9}(z) \tilde{\psi}^{\mu}(\bar{z}) e^{i k_{\rho} X^{\rho}(z, \bar{z})} e^{-\tilde{\phi}(\bar{z})} \\
& i\left(\frac{2}{\alpha^{\prime}}\right)^{1 / 2} \frac{1}{\sqrt{2 \pi R}} e_{99} \partial X_{L}^{9}(z) \tilde{\psi}^{9}(\bar{z}) e^{i k_{\rho} X^{\rho}(z, \bar{z})} e^{-\tilde{\phi}(\bar{z})}(3 .)
\end{aligned}
$$


with $\mu=0, \ldots, 8$. These operators need to be normalized by choosing polarization tensors appropriately. We take $e_{\mu \nu} e^{\mu \nu}=1$ as well as $e_{99} e^{99}=1$. For the radion, we can choose $e_{99}=1$. For a Kaluza-Klein vector field associated to the metric for instance, we can pick $e_{9 \mu}=\frac{1}{\sqrt{2}} e_{\mu}=e_{\mu 9}$ and $e_{\mu} e^{\mu}=1$. To simplify our notation we will refer to the Kaluza-Klein origin of these fields, and organize parts of our calculation as a ten-dimensional one. For large parts of the calculation, we can use ten-dimensional indices $A=0,1, \ldots, 9$ and write the vertex operators as:

$$
\begin{aligned}
& V_{T+}^{-1}(z, \bar{z})=\frac{1}{\sqrt{2 \pi R}} e^{i k_{A, L}^{+} X^{A}(z)+i k_{A, R}^{+} X^{A}(\bar{z})} e^{-\tilde{\phi}(\bar{z})}, \\
& V_{T-}^{-1}(z, \bar{z})=\frac{1}{\sqrt{2 \pi R}} e^{i k_{A, L}^{-} X^{A}(z)+i k_{A, R}^{-} X^{A}(\bar{z})} e^{-\tilde{\phi}(\bar{z})}, \\
& V_{g}^{-1}(z, \bar{z})=i\left(\frac{2}{\alpha^{\prime}}\right)^{1 / 2} \frac{1}{\sqrt{2 \pi R}} e_{A B} \partial X_{L}^{A}(z) \tilde{\psi}^{B}(\bar{z}) e^{i k_{A, L}^{g} X^{A}(z)+i k_{A, R}^{g} X^{A}(\bar{z})}
\end{aligned}
$$

with

$$
\begin{aligned}
& k_{A, L / R}^{+}=\left(k_{\mu}, k_{9, L / R}\right) \\
& k_{A, L / R}^{-}=\left(k_{\mu},-k_{9, L / R}\right) \\
& k_{A, L / R}^{g}=\left(k_{\mu}, 0\right)
\end{aligned}
$$

Note that the gravitational vertex operator $V_{g}^{-1}(z, \bar{z})$ in equation (3.3) is a sum of the four operators listed in equation (3.2). In the following we will not always write the superscripts ,,$+- g$. Which momentum we mean should be clear from the context. We will also need the tachyon vertex operators in the zero picture:

$$
\begin{aligned}
& V_{T+}^{0}(z, \bar{z})=-\left(\frac{\alpha^{\prime}}{2}\right)^{1 / 2} \frac{1}{\sqrt{2 \pi R}} \tilde{\psi}(\bar{z}) \cdot k_{R}^{+} e^{i k_{A, L}^{+} X^{A}(z)+i k_{A, R}^{+} X^{A}(\bar{z})} \\
& V_{T-}^{0}(z, \bar{z})=\left(\frac{\alpha^{\prime}}{2}\right)^{1 / 2} \frac{1}{\sqrt{2 \pi R}} \tilde{\psi}(\bar{z}) \cdot k_{R}^{-} e^{i k_{A, L}^{-} X^{A}(z)+i k_{A, R}^{-} X^{A}(\bar{z})}
\end{aligned}
$$

The dot product refers to the ten-dimensional space. We note that the vertex operator is normalized correctly since $k_{R}^{ \pm 2}=\frac{2}{\alpha^{\prime}}$. The relative sign in equations (3.5) makes sure that the two-point function between these operators is positive. We have now enumerated all vertex operators necessary to perform the scattering amplitude calculations.

\subsection{Amplitudes}

The calculation of the scattering amplitudes proceeds in standard fashion. In this section, we summarize the results of these calculations.

\subsubsection{Tachyon-tachyon-gravitational three-point amplitude}

The three-point amplitudes feature two operators in the minus one picture and one operator in the zero picture. The positions of the three vertex operators can be gauge fixed, and 
give rise to ghost operator insertions. The resulting amplitude is:

$$
\begin{aligned}
\mathcal{A}_{T^{+} T^{-} g}= & g_{c} g_{T}^{2}\left\langle c \tilde{c} V_{T+}^{0}\left(z_{1}, \bar{z}_{1}\right) c \tilde{c} V_{T-}^{-1}\left(z_{2}, \bar{z}_{2}\right) c \tilde{c} V_{g}^{-1}\left(z_{3}, \bar{z}_{3}\right)\right\rangle \\
= & i \pi \alpha^{\prime} R C_{S_{2}} g_{c} g_{T}^{2}(2 \pi)^{9} \delta^{9}\left(\sum_{i=1}^{3} k_{i}^{\mu}\right) e_{3} A B k_{1 L}^{A+} k_{2 R}^{B-} \\
= & i \pi \alpha^{\prime} R C_{S_{2}} g_{c} g_{T}^{2}(2 \pi)^{9} \delta^{9}\left(\sum_{i=1}^{3} k_{i}^{\mu}\right) \\
& \times\left(e_{3 \mu \nu} k_{1}^{\mu} k_{2}^{\nu}-e_{3 \mu 9} k_{1}^{\mu} k_{R}^{9}+e_{3}{ }_{9 \mu} k_{L}^{9} k_{2}^{\mu}-e_{399} k_{L}^{9} k_{R}^{9}\right) .
\end{aligned}
$$

The overall factor $C_{S_{2}}$ is an overall normalization factor consisting of the normalization of the $X^{A}, \psi^{A}$ matter and $\tilde{\phi}$ ghost path integrals. We will fix it by demanding unitarity [12]. To derive the amplitude we used that the external particles are on-shell as well as the fact that pure gauge modes decouple. Since the graviton couples universally to tachyons, we expect the tachyon-tachyon-graviton coupling to be the same as in bosonic string theory, and it is [13].

\subsubsection{Tachyon four-point amplitude}

The four-point tachyon amplitude with two negatively charged and two positively charged tachyons features two zero and two minus one picture operators. We order them as $\mathcal{A}_{T^{4}}=$ $\left\langle T^{+}\left(k_{1}\right) T^{-}\left(k_{2}\right) T^{+}\left(k_{3}\right) T^{-}\left(k_{4}\right)\right\rangle$. We integrate over the position of one of them, and the integral gives rise to a ratio of $\Gamma$-functions:

$$
\begin{aligned}
\mathcal{A}_{T^{4}}= & -i(2 \pi)^{2} C_{S_{2}} R\left(g_{T}\right)^{4}(2 \pi)^{9} \delta^{9}\left(\sum_{i=1}^{4} k_{i}^{\mu}\right) \\
& \times \frac{\Gamma\left(-1-\frac{\alpha^{\prime}}{4} s\right) \Gamma\left(-1-\frac{\alpha^{\prime}}{4} t+\alpha^{\prime} k_{9, L}^{2}\right) \Gamma\left(-1-\frac{\alpha^{\prime}}{4} u\right)}{\Gamma\left(1+\frac{\alpha^{\prime}}{4} s\right) \Gamma\left(3+\frac{\alpha^{\prime}}{4} t-\alpha^{\prime} k_{9, L}^{2}\right) \Gamma\left(1+\frac{\alpha^{\prime}}{4} u\right)} .
\end{aligned}
$$

The amplitude has the expected pole structure:

- s-channel poles at $\alpha^{\prime} s=0,4,8, \ldots$

- t-channel poles at $\alpha^{\prime} t=4\left(-1+\alpha^{\prime} k_{L, 9}^{2}\right)=4\left(1+\alpha^{\prime} k_{R, 9}^{2}\right), \ldots$

- u-channel poles at $\alpha^{\prime} u=0,4,8, \ldots$

The amplitude is symmetric between the $s$ and $u$-channel. The lowest mass states in these channels are the modes arising from the ten-dimensional metric, dilaton and antisymmetric tensor. In the $t$-channel, there can be no massless exchange, due to momentum and winding number conservation.

\subsubsection{Unitarity and normalization}

To compute the constant $C_{S_{2}}$, we use the unitarity relation which requires that a pole in the s-channel (say) is indeed given by the corresponding particle being exchanged through 
cubic vertices. We should therefore demand that:

$$
\begin{aligned}
& \left.\mathcal{A}_{T^{4}}\right|_{s=0}=-\frac{i(2 \pi)^{9} \pi^{2} R C_{S_{2}}\left(g_{T}^{9}\right)^{4}\left(4+\alpha^{\prime} u\right)\left(8+\alpha^{\prime} u\right)}{\alpha^{\prime} s}+\ldots \\
& \quad=i \int \frac{d^{9} k}{(2 \pi)^{9}} \frac{\mathcal{A}_{T+T-G} \mathcal{A}_{T+T-G}}{s}=-\frac{i(2 \pi)^{9} \pi^{2} R^{2} C_{S_{2}}^{2}\left(g_{c}^{9}\right)^{2}\left(g_{T}^{9}\right)^{4}\left(4+\alpha^{\prime} u\right)\left(8+\alpha^{\prime} u\right)}{4 s}+\ldots
\end{aligned}
$$

which fixes the constant to be $[13,14]$ :

$$
C_{S_{2}}=\frac{4}{\alpha^{\prime} g_{c}^{2} R}=\frac{8 \pi}{\alpha^{\prime}\left(g_{c}^{10}\right)^{2}}
$$

\subsubsection{Tachyon-Tachyon-Gravitational-Gravitational four-point amplitude}

As a cross-check on our understanding of the calculation of the amplitudes, we wish to confirm that each vertex operator should be accompanied by one and the same coupling constant. To that end, we compute the two tachyon two graviton four-point amplitude and find:

$$
\begin{aligned}
\mathcal{A}_{T^{+} T^{-} g g}= & -i(2 \pi)^{11} R C_{S_{2}} g_{c}^{2} g_{T}^{2} \delta^{9}\left(\sum_{i=1}^{4} k_{i}^{\mu}\right) e_{3 A B} e_{4 C D} \times \\
& \times\left\{\alpha^{\prime}(f(u)-1) k_{1 L}^{A}\left((f(t)-1) k_{1 L}^{C}+f(u) k_{2 L}^{C}\right)\right. \\
& \left.\times \alpha^{\prime}(f(t)-1) k_{2 L}^{A}\left(f(t) k_{1 L}^{C}+(f(u)-1) k_{2 L}^{C}\right)+2(f(t)-1)(f(u)-1) \eta^{A C}\right\} \\
& \times\left\{\alpha^{\prime}(f(t)-1) k_{2 R}^{B} k_{1 R}^{D}+\alpha^{\prime}(f(u)-1) k_{1 R}^{B} k_{2 R}^{D}+2(f(t)-1)(f(u)-1) \eta^{B D}\right\} \\
& \times \frac{\Gamma(1-f(t)-f(u)) \Gamma(-1+f(t)) \Gamma(-1+f(u))}{\Gamma(-1+f(t)+f(u)) \Gamma(2-f(t)) \Gamma(2-f(u))},
\end{aligned}
$$

where the function $f$ is defined by the formula:

$$
f(x)=-\frac{\alpha^{\prime}}{4}\left(x+k_{L, 9}^{2}\right) .
$$

The t-channel pole structure is:

$$
\begin{aligned}
\left.\mathcal{A}_{T^{+} T^{-} g g}\right|_{t=k_{L, 9}^{2}-4 / \alpha^{\prime}}= & -\frac{4 i(2 \pi)^{11} \alpha^{\prime} R C_{S_{2}} g_{c}^{2} g_{T}^{2} e_{4 C D} k_{1 L}^{A} k_{1 R}^{B} e_{3 A B} k_{2 L}^{C} k_{2 R}^{D}}{t-k_{L, 9}^{2}+4 / \alpha^{\prime}} \\
& + \text { terms analytic at } k^{2}=-k_{L, 9}^{2}+4 / \alpha^{\prime}
\end{aligned}
$$

This is reproduced by tachyon exchange in the $t$-channel:

$$
i \int \frac{d^{9} k}{(2 \pi)^{9}} \frac{\mathcal{A}_{T^{+} T^{-} g} \mathcal{A}_{T^{+} T^{-} g}}{t-k_{L, 9}^{2}+4 / \alpha^{\prime}}=-\frac{i(2 \pi)^{11} \alpha^{\prime 2} R^{2} C_{S_{2}}^{2} g_{c}^{2} g_{T}^{4} e_{3 A B} k_{1 L}^{A} k_{1 R}^{B} e_{4 C D} k_{2 L}^{C} k_{2 R}^{D}}{t-k_{L, 9}^{2}+4 / \alpha^{\prime}},
$$

on the condition that we have $g_{c}=g_{T}$, as expected in any string theory. 


\subsubsection{Summary}

The three amplitudes can be simplified, using that $g=g_{c}=g_{T}$ and using the value for the constant $C_{S_{2}}$ :

$$
\begin{aligned}
\mathcal{A}_{T^{+} T^{-} g}= & 4 i \pi g(2 \pi)^{9} \delta^{9}\left(\sum_{i=1}^{3} k_{i}^{\mu}\right) e_{3 A B} k_{1 L}^{A+} k_{2 R}^{B-} \\
\mathcal{A}_{T^{4}}= & -\frac{16 i \pi^{2} g^{2}}{\alpha^{\prime}}(2 \pi)^{9} \delta^{9}\left(\sum_{i=1}^{4} k_{i}^{\mu}\right) \\
& \times \frac{\Gamma\left(-1-\frac{\alpha^{\prime}}{4} s\right) \Gamma\left(-1-\frac{\alpha^{\prime}}{4} t+\alpha^{\prime} k_{9, L}^{2}\right) \Gamma\left(-1-\frac{\alpha^{\prime}}{4} u\right)}{\Gamma\left(1+\frac{\alpha^{\prime}}{4} s\right) \Gamma\left(3+\frac{\alpha^{\prime}}{4} t-\alpha^{\prime} k_{9, L}^{2}\right) \Gamma\left(1+\frac{\alpha^{\prime}}{4} u\right)}, \\
\mathcal{A}_{T^{+} T^{-} g^{2}}= & -\frac{16 i \pi^{2} g^{2}}{\alpha^{\prime}}(2 \pi)^{9} \delta^{9}\left(\sum_{i=1}^{4} k_{i}^{\mu}\right) e_{3 A B} e_{4 C D} \\
& \times\left(\alpha^{\prime}(f(u)-1) k_{1 L}^{A}\left((f(t)-1) k_{1 L}^{C}+f(u) k_{2 L}^{C}\right)\right. \\
& \left.+\alpha^{\prime}(f(t)-1) k_{2 L}^{A}\left(f(t) k_{1 L}^{C}+(f(u)-1) k_{2 L}^{C}\right)+2(f(t)-1)(f(u)-1) \eta^{A C}\right) \\
& \times\left(\alpha^{\prime}(f(t)-1) k_{2 R}^{B} k_{1 R}^{D}+\alpha^{\prime}(f(u)-1) k_{1 R}^{B} k_{2 R}^{D}+2(f(t)-1)(f(u)-1) \eta^{B D}\right) \\
& \times \frac{\Gamma(1-f(t)-f(u)) \Gamma(-1+f(t)) \Gamma(-1+f(u))}{\Gamma(-1+f(t)+f(u)) \Gamma(2-f(t)) \Gamma(2-f(u))} .
\end{aligned}
$$

The above amplitudes are valid at any radius.

\section{The effective action}

In this section, we will use the on-shell scattering amplitudes computed in section 3 to fix terms in the low-energy effective action. We start out by analyzing the effective action at the Hagedorn point(s). We will fix cubic and quartic couplings using the scattering amplitudes.

\subsection{The action}

At the Hagedorn radius, we have the low-energy excitations arising from Kaluza-Klein reduction from ten dimensions, supplemented with the massless complex tachyon. We can obtain terms in the effective Lagrangian through dimensional reduction. The reduced action will contain a graviton, an anti-symmetric tensor, two scalar fields, and two Maxwell gauge fields. We then need to couple in the tachyon. We ignore the $\operatorname{Spin}(32) / \mathbb{Z}_{2}$ gauge theory sector. We follow the conventions of [13] and write the ten-dimensional string metric as:

$$
d s^{2}=g_{\mu \nu} d x^{\mu} d x^{\nu}+g_{99}\left(d x^{9}+A_{\mu}^{g} d x^{\mu}\right)^{2},
$$


and we will also write the nine-nine component of the metric in terms of a radion field $\sigma$, $g_{99}=e^{\sigma}$. The vector field $A_{\mu}^{g}$ is the metric Kaluza-Klein gauge field. In this section, we take the ninth direction to be at the Hagedorn radius $x^{9} \equiv x^{9}+2 \pi R_{H}$.

In the Einstein frame defined by $g_{\mu \nu}^{E}=e^{-\frac{4 \Phi_{9}}{D-2}} g_{\mu \nu}$ (and $D=9$ ), we have the following dimensionally reduced action:

$$
\begin{aligned}
S^{9(E)}= & \frac{1}{2 \kappa_{9}^{2}} \int d^{8+1} \sqrt{-g_{9}^{E}}\left(R^{(9)}-\frac{4}{D-2} \partial_{\mu} \Phi_{9} \partial^{\mu} \Phi_{9}-\partial_{\mu} \sigma \partial^{\mu} \sigma-\frac{1}{4} e^{2 \sigma} e^{-\frac{4 \Phi_{9}}{D-2}} F_{\mu \nu}^{g} F^{g \mu \nu}\right. \\
& \left.-\frac{1}{4} e^{-2 \sigma} e^{-\frac{4 \Phi_{9}}{D-2}} F_{\mu \nu}^{b} F^{b \mu \nu}-2 \kappa_{9}^{2} D_{\mu} T^{+} D^{\mu} T^{-}-2 \kappa_{9}^{2} e^{\frac{4 \Phi_{9}}{D-2}} V(\sigma,|T|)\right) .
\end{aligned}
$$

We added in a canonically normalized kinetic and potential term for the complex tachyon. By virtue of its momentum and winding charge, the tachyon is charged under both KaluzaKlein gauge fields, and the kinetic term features gauge covariant derivatives:

$$
D_{\mu}=\partial_{\mu}+i q^{g} A_{\mu}^{g}+i q^{b} A_{\mu}^{b} .
$$

We can fix part of the potential by using our knowledge of the tachyon mass squared as a function of the radion vacuum expectation value. We therefore must have the following interaction term between the radion and the tachyon:

$$
\begin{aligned}
S_{m^{2}} & =-\int d^{8+1} x \sqrt{-g} m^{2}\left(R=e^{\sigma} R_{H}\right) T^{+} T^{-} \\
& =-\int d^{8+1} x \sqrt{-g}\left(-\frac{4}{\alpha^{\prime}}+\left(\frac{1}{2 R}+\frac{R}{\alpha^{\prime}}\right)^{2}\right) T^{+} T^{-} \\
& =-\int d^{8+1} x \sqrt{-g}\left(-\frac{4}{\alpha^{\prime}}+\left(\frac{e^{-\sigma}}{2 R_{H}}+\frac{e^{\sigma} R_{H}}{\alpha^{\prime}}\right)^{2}\right) T^{+} T^{-} .
\end{aligned}
$$

We parameterize the potential term quartic in the tachyon as follows:

$$
S_{\text {quartic }}=-\int d^{8+1} \frac{\lambda}{4}|T|^{4} .
$$

\subsection{Expansion of the action around the flat background}

We expand the action around the flat background, with constant dilaton, the radius at the Hagedorn point, zero tachyon vacuum expectation value, etcetera. We will parameterize the fluctuation in the Einstein metric by: $g_{\mu \nu}=\eta_{\mu \nu}-2 \kappa_{9} h_{\mu \nu}$ and will use the field variable to parameterize the fluctuations for other fields. As a result of expanding the effective action to cubic order, we find:

$$
\begin{aligned}
& S^{9 \quad \mathrm{cub}}=\int d^{8+1} x\left(-\frac{1}{2} \partial_{\mu} h_{\nu \rho} \partial^{\mu} h^{\nu \rho}-2 \kappa_{9}\left(h^{\mu \nu}-\frac{1}{2} h_{\rho}{ }^{\rho} \eta^{\mu \nu}\right) \partial_{\mu} T^{+} \partial_{\nu} T^{-}\right. \\
& +\frac{1}{2 \kappa_{9}^{2}}\left(-\frac{4}{D-2} \partial_{\mu} \Phi_{9} \partial^{\mu} \Phi_{9}-\partial_{\mu} \sigma \partial^{\mu} \sigma-\frac{1}{4} F_{\mu \nu}^{g} F^{g \mu \nu}-\frac{1}{4} F_{\mu \nu}^{b} F^{b \mu \nu}\right) \\
& \left.-\frac{i}{\kappa_{9}^{2}}\left(q^{g} A_{\mu}^{g}+q^{b} A_{\mu}^{b}\right) T^{[+} \partial^{\mu} T^{-]}-\frac{4 \sqrt{2}}{\alpha^{\prime}} \sigma T^{+} T^{-}\right) .
\end{aligned}
$$


The normalization of the kinetic term of the gauge field and the Kaluza-Klein momenta of the tachyon imply that we must identify the charges in these equations as the momentum and winding around the Kaluza-Klein circle:

$$
q^{g}=\frac{1}{2}\left(k_{L, 9}+k_{R, 9}\right)=\frac{n}{R}, \quad q^{b}=\frac{1}{2}\left(k_{L, 9}-k_{R, 9}\right)=\frac{w R}{\alpha^{\prime}} .
$$

In the following, we will sometimes refer to the canonically normalized radion $\sigma^{\prime}=\frac{\sigma}{\kappa_{9}}$ and the canonically normalized vector bosons $A_{\mu}^{\prime}=\frac{1}{\sqrt{2} \kappa_{9}} A_{\mu}$.

\subsection{Cubic couplings}

We now check the coefficients of the cubic couplings in the effective action against the string theory three-point amplitudes evaluated at low energy and at the Hagedorn point $R_{H} / \sqrt{\alpha^{\prime}}=1+1 / \sqrt{2}$. To compare the three-point amplitudes in equation (3.13) to the effective action, we rewrite them using left- and right-momentum conservation, as well as the Ward identities. We also use the values of the ten-dimensional momenta:

$$
\begin{array}{cc}
k_{1 L}^{A}=\left(k_{1}^{\mu}, k_{L}^{9}\right), & k_{2 L}^{A}=\left(k_{2}^{\mu},-k_{L}^{9}\right), \\
k_{1 R}^{A}=\left(k_{1}^{\mu}, k_{R}^{9}\right), & k_{2 R}^{A}=\left(k_{2}^{\mu},-k_{R}^{9}\right), \\
k_{3}^{A}=\left(k_{3}^{\mu}, 0\right), &
\end{array}
$$

and we obtain

$$
\begin{aligned}
& 4 i \pi g_{c} e_{3 A B} k_{1 L}^{A} k_{2 R}^{B}=2 i \pi g_{c} e_{3 A B}\left(k_{1 L}^{A} k_{2 R}^{B}+k_{2 L}^{A} k_{1 R}^{B}\right) \\
& \quad=2 i \pi g_{c}\left(e_{3 \mu \nu}\left(k_{1}^{\mu} k_{2}^{\nu}+k_{2}^{\mu} k_{1}^{\nu}\right)+\left(k_{2}^{\mu}-k_{1}^{\mu}\right)\left(k_{L}^{9} e_{3} 9 \mu+k_{R}^{9} e_{3 \mu 9}\right)-2 e_{3} 99 k_{L}^{9} k_{R}^{9}\right) .
\end{aligned}
$$

To read the effective action terms from the amplitude, we use the correspondence between the Fourier coefficients of the fields in the effective action and the momentum dependent polarization tensors used in the string amplitude computation:

$$
\begin{aligned}
e_{(\mu \nu)}-\frac{1}{D-2} e_{\rho}^{\rho} \delta_{\mu \nu}^{\perp} & \rightarrow h_{\mu \nu}(k) \\
e_{[\mu \nu]} & \rightarrow b_{\mu \nu}(k) \\
\frac{1}{\sqrt{D-2}} e_{\mu}^{\mu} & \rightarrow \Phi_{9}^{\prime}(k) \\
\frac{1}{\sqrt{2}}\left(e_{9 \mu} \pm e_{\mu 9}\right) & \rightarrow A_{\mu}^{\prime g / b}(k) \\
e_{99} & \rightarrow \sigma^{\prime}(k)
\end{aligned}
$$

with $\delta_{\mu \nu}^{\perp}=\eta_{\mu \nu}-k_{\mu} n_{\nu}-n_{\mu} k_{\nu}$ and where a reference momentum $n$ is chosen such that it satisfies $n^{2}=0$ and $k \cdot n=1$. The normalized vertex operators correspond to fields $\Phi_{9}^{\prime}$, $A_{\mu}^{\prime g / b}$ and $\sigma^{\prime}$ that have canonically normalized kinetic terms. 
Next, we note that we have the following matches between the cubic couplings and terms in the effective action:

$$
\begin{array}{rlrr}
4 \pi i g e_{3 \mu \nu} k_{1}^{\mu} k_{2}^{\nu} & \rightarrow & -2 \kappa_{9} h^{\mu \nu} \partial_{\mu} T^{+} \partial_{\nu} T^{-} \\
\pi i g\left(k_{L, 9} \pm k_{R, 9}\right)\left(e_{9 \mu} \pm e_{\mu 9}\right)\left(k_{2}^{\mu}-k_{1}^{\mu}\right) & \rightarrow & -2 \sqrt{2} i \kappa_{9} q^{g / b} A^{\prime} g / b \mu_{T} T^{[+} \partial_{\mu} T^{-]} \\
-4 \pi i g k_{L, 9} k_{R, 9} & \rightarrow & -2 \kappa_{9} k_{L, 9} k_{R, 9} \sigma^{\prime} T^{+} T^{-}
\end{array}
$$

if we identify

$$
2 \pi g=\kappa_{9}
$$

Our conventions are such that the cubic three-point amplitudes for three particles corresponding to the fields $A_{i}$ are given by:

$$
\left\langle A_{1}\left(k_{1}\right) A_{2}\left(k_{3}\right) A_{3}\left(k_{3}\right)\right\rangle_{c u b i c}=\left.\frac{i \delta^{3} S}{\delta A_{1}\left(k_{1}\right) \delta A_{2}\left(k_{2}\right) \delta A_{3}\left(k_{3}\right)}\right|_{A_{i}=0},
$$

which applied to the effective action terms gives the low-energy limit of the three-point string amplitudes.

\subsection{Remark}

We note that the couplings $\sigma \partial_{\mu} T^{+} \partial^{\mu} T^{-}$and $\phi \partial_{\mu} T^{+} \partial^{\mu} T^{-}$do not contribute to the onshell three-point amplitude at the Hagedorn point. We can fix them through the following requirement and reasoning. Firstly, we assumed that the tachyon kinetic term is canonical at any radius. Thus, we chose a basis of fields such that the term $\sigma \partial_{\mu} T^{+} \partial^{\mu} T^{-}$is absent from the Lagrangian. Otherwise, a non-zero vacuum expectation value for the radion $\sigma$ would lead to a non-canonical kinetic term for the tachyon. Secondly, by the universality of the dependence of the string effective action on the dilaton, we note that after going to Einstein frame, the tachyon kinetic term is dilaton independent. Thus, the term $\phi \partial_{\mu} T^{+} \partial^{\mu} T^{-}$is also absent from the Lagrangian.

\subsection{A quartic coupling}

In this subsection, we want to determine the quartic term in the tachyon potential. The tachyon four-point amplitude in string theory, expanded under the assumptions $u \alpha^{\prime} \ll$ $1, s \alpha^{\prime} \ll 1$ gives rise to poles, constant terms and terms of higher order in the momenta:

$$
\begin{aligned}
\mathcal{A}_{T^{4}} & \approx-\frac{32 i \kappa_{9}^{2}}{\alpha^{\prime 2}}\left(\frac{3}{4} \alpha^{\prime}+\frac{1}{s}\left(1+\frac{\alpha^{\prime}}{4} u\right)\left(1+\frac{\alpha^{\prime}}{8} u\right)+\frac{1}{u}\left(1+\frac{\alpha^{\prime}}{4} s\right)\left(1+\frac{\alpha^{\prime}}{8} s\right)\right)+\ldots \\
& \approx-i \kappa_{9}^{2}\left(\frac{24}{\alpha^{\prime}}+\left(\frac{u^{2}}{s}+\frac{s^{2}}{u}\right)+\frac{12}{\alpha^{\prime}}\left(\frac{u}{s}+\frac{s}{u}\right)+\frac{32}{\alpha^{\prime 2}}\left(\frac{1}{s}+\frac{1}{u}\right)+\ldots\right) .
\end{aligned}
$$

As a cross-check on the overall sign, we note that the graviton exchange is as in the VirasoroShapiro amplitude in bosonic string theory [13]. The pole contributions of the amplitude correspond to the exchange of massless particles. These exchange contributions can also give rise to constant contributions that we need to subtract in order to isolate the contribution from the quartic contact term. Above we have fixed a choice of fields and associated cubic couplings, so that the exchange contribution is now fixed. Let's compute it. 


\subsubsection{Massless exchange contributions to the four-point tachyon amplitude}

The massless states that can be exchanged by the tachyons are the graviton, the radion, the dilaton and the two gauge bosons. We compute the amplitude for the exchange diagrams using the expression

$$
\mathcal{A}_{A_{1} A_{2} \rightarrow B_{1} B_{2}}=i \frac{\delta^{3} S}{\delta A_{1}\left(k_{1}\right) \delta A_{2}\left(k_{2}\right) \delta A_{3}\left(k_{3}\right)} \Delta_{A_{3} A_{3}}^{-1} i \frac{\delta^{3} S}{\delta A_{3}\left(k_{4}\right) \delta B_{1}\left(k_{5}\right) \delta B_{2}\left(k_{6}\right)}
$$

for the exchange amplitude, where $\Delta^{-1}$ is the propagator of the exchanged state. Due to the fact that both the $\sigma \partial_{\mu} T^{+} \partial^{\mu} T^{-}$and the $\Phi_{9} \partial_{\mu} T^{+} \partial^{\mu} T^{-}$term are absent in our effective Lagrangian, we can concentrate on the non-derivative cubic terms in the action for the radion and the dilaton. Due to the fact that the tachyon is massless, there is moreover no $\Phi_{9} T^{+} T^{-}$term in the action. We therefore can concentrate on the exchange of the graviton, the radion and the two gauge fields.

- We compute the graviton exchange in the harmonic gauge using the propagator:

$$
\Delta_{\mu \nu, \rho \sigma}^{-1}=-i\left(\frac{1}{2} \eta_{\mu \rho} \eta_{\nu \sigma}+\frac{1}{2} \eta_{\mu \sigma} \eta_{\nu \rho}-\frac{1}{D-2} \eta_{\mu \nu} \eta_{\rho \sigma}\right) \frac{1}{k^{2}} .
$$

The $s$-channel contribution is:

$$
\begin{aligned}
\mathcal{A}_{T^{+} T^{-} \rightarrow T^{+} T^{-}}^{\text {s-chanel }} & =\left(2 i \kappa_{9}\left(k_{1}^{\mu} k_{2}^{\nu}-\frac{1}{2} \eta^{\mu \nu} k_{1} \cdot k_{2}\right)\right) \Delta_{\mu \nu, \rho \sigma}^{-1}\left(2 i \kappa_{9}\left(k_{3}^{\rho} k_{4}^{\sigma}-\frac{1}{2} \eta^{\rho \sigma} k_{3} \cdot k_{4}\right)\right) \\
& =-i \kappa_{9}^{2}\left(\frac{u^{2}}{s}+u\right) .
\end{aligned}
$$

Summing over $s-$ and $u$-channels gives:

$$
\mathcal{A}_{T^{+} T^{-} \rightarrow T^{+} T^{-}}^{\text {graviton exchang }}=-i \kappa_{9}^{2}\left(\frac{u^{2}}{s}+\frac{s^{2}}{u}+s+u\right) \text {. }
$$

- The terms in the action relevant to the normalized radion exchange are:

$$
S=-\frac{1}{2} \int d^{8+1} x\left(\partial_{\mu} \sigma^{\prime} \partial^{\mu} \sigma^{\prime}+\kappa_{9} \frac{8 \sqrt{2}}{\alpha^{\prime}} \sigma^{\prime} T^{+} T^{-}\right) .
$$

The radion propagator is $\Delta_{\sigma^{\prime}}=-\frac{i}{k^{2}}$, such that we obtain the radion exchange contribution to the tachyon four-point amplitude:

$$
\mathcal{A}_{T^{+} T^{-} \rightarrow T^{+} T^{-}}^{\text {radion exchange }}=-\frac{32 i \kappa_{9}^{2}}{\alpha^{\prime 2}}\left(\frac{1}{s}+\frac{1}{u}\right) .
$$

- Both the metric gauge field $A^{g}$ and the anti-symmetric tensor gauge field $A^{b}$ are exchanged. Since the terms in the action are the same up to the charge, we find an exchange contribution proportional to the respective charges squared. The canonically normalized fields have an action equal to:

$$
\int d^{8+1} x\left(-\frac{1}{4} F_{\mu \nu}^{\prime g} F^{\prime g \mu \nu}-\sqrt{2} i \kappa_{9} q^{g} A_{\mu}^{\prime g}\left(T^{+} \partial^{\mu} T^{-}-T^{-} \partial^{\mu} T^{+}\right)\right) .
$$


Using the propagator

$$
\Delta_{\mu \nu}^{-1}=-\frac{i \eta_{\mu \nu}}{k^{2}}
$$

we then find the total contribution of both gauge bosons to the tachyon four-point amplitude summed over $s$ - and $u$-channel to be:

$$
\mathcal{A}_{T^{+} T^{-} \rightarrow T^{+} T^{-}}^{\text {boson exchange }}=-2 i \kappa_{9}^{2}\left(q_{g}^{2}+q_{b}^{2}\right)\left(\frac{2 s}{u}+\frac{2 u}{s}+2\right)=-i \kappa_{9}^{2} \frac{12}{\alpha^{\prime}}\left(\frac{s}{u}+\frac{u}{s}+1\right),
$$

where in the last step we inserted the value of the charges at the Hagedorn radius $q^{g / b}=\frac{1}{\sqrt{\alpha^{\prime}}}\left(1 \pm \frac{1}{\sqrt{2}}\right)$.

- In summary, the sum of the exchange contributions of all massless states in both channels are:

$$
\mathcal{A}_{T^{+} T^{-} \rightarrow T^{+} T^{-}}^{\text {all }}=-i \kappa_{9}^{2}\left(\left(\frac{u^{2}}{s}+\frac{s^{2}}{u}+s+u\right)+\frac{32}{\alpha^{\prime 2}}\left(\frac{1}{s}+\frac{1}{u}\right)+\frac{12}{\alpha^{\prime}}\left(\frac{s}{u}+\frac{u}{s}+1\right)\right) .
$$

The contributions match the terms in the expansion of the amplitude $A_{T^{4}}$ in equation (4.14) near small momenta $u \alpha^{\prime} \ll 1, s \alpha^{\prime} \ll 1$. Note that the gauge boson exchange gives rise to a constant term. Indeed, the pole can be cancelled by the two cubic one-derivative interactions. We also remark that the scalars don't give rise to a constant term because of the absence of cubic one-derivative couplings.

\subsubsection{The quartic term in the tachyon potential}

The contact contribution to the amplitude arising from the quartic term in the effective action for the tachyon is given by:

$$
-i \lambda
$$

To obtain the value of the coupling constant $\lambda$ we subtract the contributions from the exchange diagrams in equation (4.24) from the expansion of the amplitude $A_{T^{4}}$ in equation (4.14) near small momenta. We find:

$$
\lambda=\frac{12 \kappa_{9}^{2}}{\alpha^{\prime}} .
$$

The potential due to the contact term is a repulsive delta-function potential.

\subsubsection{Remark}

We believe it is an interesting result to have determined the quartic tachyon potential in our choice of field basis. For the order of the phase transition at the Hagedorn point though, the cubic interaction between the radion and the tachyon is sufficient to argue it is of first order [1]. One reason to nevertheless go through this elaborate determination of the quartic potential at the Hagedorn point is that it can be determined rigorously at this point. We will attempt to draw lessons from this exercise in the next, more exploratory section. 


\section{The effective action at smaller radii}

In this section, we want to speculate about what happens at smaller radii, and in particular at the most symmetric point, namely the self-dual radius. We face a generic problem that plagues our understanding of the physics at the self-dual radius, which is that on-shell, the tachyon momentum will be of order one over the string scale, thus rendering invalid the derivative expansion in the effective action. Only for very short time scales would one be able to trust the effective action near flat space. In this section, we therefore concentrate on enumerating some generic expected properties of the effective action, and we will explore one avenue to get a handle on the physics at small radii.

\subsection{The effective action at the self-dual radius}

At self-dual radius, we will have an enhanced $\mathrm{SU}(2)_{R} \times \mathrm{U}(1)_{L} \times \operatorname{Spin}(32) / \mathbb{Z}_{2}$ gauge symmetry. The radion joins a $(3,0,1)$ triplet, and the $\operatorname{Spin}(32) / \mathbb{Z}_{2}$ adjoint scalar also becomes part of a triplet. The $\mathrm{SU}(2)_{R}$ triplet will presumably give rise to a quartic commutator square potential. The triplet and the tachyon will also give rise to a quartic term, and we may expect a potential in terms of the tachyon only as well. We can also have a commutator squared term for a mix of the two $\mathrm{SU}(2)_{R}$ triplets. We note that the cubic potential term $\sigma T^{+} T^{-}$is absent at the self-dual radius, since the self-dual radius corresponds to the minimal value of the tachyon mass squared.

For concreteness, let us focus on the term in the potential that depends on the complex tachyon only. For starters, we note that the tachyon vertex operator in the zero picture has a term proportional to the space-time momentum, and a term proportional to the chiral compact momentum $p_{R}$ on the supersymmetric side of the heterotic string. The momentum $p_{R}$ is zero at the self-dual point. Furthermore, the tachyon $N$-point function with $N$ larger or equal than two contains at least one zero picture tachyon vertex operator. Therefore, all the $N$-point amplitudes will be proportional to at least one factor of the space-time momentum. In fact, by Lorentz symmetry, they will be proportional to at least the space-time momentum squared. However, we cannot use this property to exclude a tachyon potential, since the integration over the positions of the tachyon vertex operators leads to extra poles in the amplitude. We will proceed differently to understand how the tachyon potential depends on the radius.

\subsection{A universal behaviour}

In fact, if we attempt to identify universal properties of the tachyon four-point amplitude at any radius, we can proceed as follows. Since the mass squared of the tachyon is radius dependent, we are tempted to eliminate it using the on-shell relation. ${ }^{2}$ After this elimination, we wind up with a four-point amplitude:

$$
\mathcal{A}_{T^{4}}=-\frac{16 i \pi^{2} g^{2}}{\alpha^{\prime}}(2 \pi)^{9} \delta^{9}\left(\sum_{i=1}^{4} k_{i}^{\mu}\right) \frac{\Gamma\left(-1-\frac{\alpha^{\prime}}{4} s\right) \Gamma\left(1+\frac{\alpha^{\prime}}{4}(s+u)\right) \Gamma\left(-1-\frac{\alpha^{\prime}}{4} u\right)}{\Gamma\left(1+\frac{\alpha^{\prime}}{4} s\right) \Gamma\left(1-\frac{\alpha^{\prime}}{4}(u+s)\right) \Gamma\left(1+\frac{\alpha^{\prime}}{4} u\right)}
$$

\footnotetext{
${ }^{2}$ Our procedure is inspired by [15].
} 
which depends on the Mandelstam invariants $s$ and $u$ only. These are the Mandelstam variables which exhibit massless poles. The only radial dependence in the amplitude is an overall factor. Since the amplitude no longer depends on the mass squared of the tachyon, we can attempt to interpret this amplitude as being off-shell. We could think of it as a function of $s, t, u$ which happens to be independent of $t$, with no kinematic constraint on the variables. A justification of this off-shell extension would have to come from a heterotic string field theory.

We now wish to venture even further, and we will attempt to identify the quartic tachyon potential term coded in this amplitude.

\subsection{Field theory exchange diagrams}

We will take a pedestrian approach here, and discuss the same field theory exchange diagrams that we already identified before, in an optimistic analogy to the effective action approach valid at the Hagedorn radius. We do take into account the change in the mass squared of the tachyons, the extra couplings this generates to the dilaton, the trace of the metric, and the change in the gauge couplings of the tachyon. We also note that the tachyon is an $\mathrm{SU}(2)_{R}$ singlet, which dictates that no new massless gauge bosons are exchanged.

The cubic tachyon coupling to the graviton, and to the dilaton acquire extra terms that can be read off from the expansion:

$$
\begin{aligned}
S^{9(E)} & =\int d^{8+1} x \sqrt{-g}\left(-\frac{1}{\kappa_{9}^{2}} \frac{2}{D-2} \partial_{\mu} \Phi_{9} \partial^{\mu} \Phi_{9}-e^{\frac{4 \Phi_{9}}{D-2}} m^{2} T^{+} T^{-}+\ldots\right) \\
& \approx \int d^{8+1} x\left(-\frac{1}{2} \partial_{\mu} \Phi_{9}^{\prime} \partial^{\mu} \Phi_{9}^{\prime}-\frac{2 m^{2}}{\sqrt{D-2}} \kappa_{9} \Phi_{9}^{\prime} T^{+} T^{-}+\kappa_{9} m^{2} h^{\mu}{ }_{\mu} T^{+} T^{-}+\ldots\right)
\end{aligned}
$$

with $\Phi_{9}^{\prime}=\frac{1}{\kappa_{9}} \frac{2}{\sqrt{D-2}} \Phi_{9}$ (and $D=9$ in our case). The cubic coupling to the dilaton and the trace of the metric are proportional as determined by the universal prefactor $e^{-2 \Phi_{9}} \sqrt{-g}$ to the potential term in the string frame. The full cubic vertices become:

$$
\begin{aligned}
V_{T^{+} T^{-} h} & =2 i \kappa_{9}\left(k_{1}^{\mu} k_{2}^{\nu}-\frac{1}{2} \eta^{\mu \nu}\left(k_{1} \cdot k_{2}-m^{2}\right)\right) \\
V_{T^{+} T^{-} \Phi_{9}^{\prime}} & =-\frac{2 i m^{2} \kappa_{9}}{\sqrt{D-2}}
\end{aligned}
$$

The graviton and the dilaton exchange then sum up to:

$$
\begin{aligned}
\mathcal{A}^{\mathrm{h}+\Phi^{\prime} \text { exchange }=} & -2 i \kappa_{9}^{2}\left(\frac{1}{s}\left(k_{1} \cdot k_{3} k_{2} \cdot k_{4}+k_{1} \cdot k_{4} k_{2} \cdot k_{3}-\left(k_{1} \cdot k_{2}-m^{2}\right)\left(k_{3} \cdot k_{4}-m^{2}\right)\right)\right. \\
& \left.+\frac{1}{u}\left(k_{1} \cdot k_{3} k_{2} \cdot k_{4}+k_{1} \cdot k_{2} k_{3} \cdot k_{4}-\left(k_{1} \cdot k_{4}-m^{2}\right)\left(k_{2} \cdot k_{3}-m^{2}\right)\right)\right) \\
= & -i \kappa_{9}^{2}\left(\frac{u^{2}}{s}+\frac{s^{2}}{u}+u+s+4 m^{2}\left(-1-\left(\frac{u}{s}+\frac{s}{u}\right)+m^{2}\left(\frac{1}{s}+\frac{1}{u}\right)\right)\right) \cdot
\end{aligned}
$$


Note that $D$-dependence drops out. The gauge boson exchange gives rise to the amplitude:

$$
\begin{aligned}
\mathcal{A}^{\text {A exchnage }} & =-4 i \kappa_{9}^{2}\left(q_{g}^{2}+q_{b}^{2}\right)\left(\frac{1}{s}\left(k_{1} \cdot k_{3}-k_{1} \cdot k_{4}\right)+\frac{1}{u}\left(k_{1} \cdot k_{3}-k_{1} \cdot k_{2}\right)\right) \\
& =-i \kappa_{9}\left(\frac{12}{\alpha^{\prime}}+4 m^{2}\right)\left(1+\frac{u}{s}+\frac{s}{u}-2 m^{2}\left(\frac{1}{s}+\frac{1}{u}\right)\right) .
\end{aligned}
$$

The radion exchange contribution becomes:

$$
\begin{aligned}
\mathcal{A}^{\sigma \text { exchnage }} & =-4 i \kappa_{9}^{2} k_{L}^{2} k_{R}^{2}\left(\frac{1}{s}+\frac{1}{u}\right)=-4 i \kappa_{9}^{2}\left(m^{2}+\frac{4}{\alpha^{\prime}}\right)\left(m^{2}+\frac{2}{\alpha^{\prime}}\right)\left(\frac{1}{s}+\frac{1}{u}\right) \\
& =-i \kappa_{9}^{2}\left(4 m^{4}+\frac{24 m^{2}}{\alpha^{\prime}}+\frac{32}{\alpha^{\prime 2}}\right)\left(\frac{1}{s}+\frac{1}{u}\right) .
\end{aligned}
$$

All exchanges summed up give:

$$
\mathcal{A}_{T^{+} T^{-} \rightarrow T^{+} T^{-}}^{\text {all exhange }}=-i \kappa_{9}^{2}\left(\left(\frac{u^{2}}{s}+\frac{s^{2}}{u}+s+u\right)+\frac{32}{\alpha^{\prime 2}}\left(\frac{1}{s}+\frac{1}{u}\right)+\frac{12}{\alpha^{\prime}}\left(\frac{s}{u}+\frac{u}{s}+1\right)\right) .
$$

We note that the mass squared dependence of the exchange diagrams drops out in the total exchange contribution. For the singular terms in the Mandelstam variables $s$ and $u$, this has to be the case, as they need to reproduce the mass squared independent singular terms in the string scattering amplitude. We find that also the constant term is independent of the mass squared in our frame.

\subsubsection{Universality}

As a consequence of the independence of the mass squared of both the amplitude and the exchange contribution as a function of the Mandelstam variables $s$ and $u$, the quartic tachyon potential contact term will remain $\lambda=12 \kappa_{9}^{2} / \alpha^{\prime}$ at all radii. One needs more information to conclude that there is a new locally stable minimum (e.g. at the self-dual radius).

\section{Conclusions and speculations}

We have reviewed the spectrum of the heterotic superstring at high temperature compared to the string scale. Equivalently, we have analyzed the theory after compactification on a circle with supersymmetry breaking periodicity conditions. We determined the coefficient of the quartic term in the tachyon potential at the Hagedorn radius. The calculation was presented in great detail, including normalized vertex operators, a unitarity check, and careful subtraction of the exchange diagrams.

We argued that a description of the physics in terms of a spontaneously broken supersymmetric theory needs to take into account winding modes that generically will not fit into supermultiplets. This phenomenon is typical of string theories. We also showed that naively extending the range of validity of the effective action to string scale regions leads us to a tachyon quartic potential that has a coefficient independent of the radius of compactification. 
If we let both the radion and the dilaton fluctuate, then Yukawa type interactions will ensure a first order phase transition. If we fix the radius at the self-dual value, and ignore the runaway dilaton, we find a quartic tachyon potential with string scale symmetry breaking minima. Massive stringy modes, higher tachyon potential terms, and higher derivative terms will then become important and they could either stabilize or destabilize the new phase.

If we take the view where we compactify a space-like circle with supersymmetry breaking periodicity conditions, then the winding tachyon is a straightforward physical excitation that can obtain a new vacuum expectation value. We find it interesting to speculate that below the Hagedorn radius, the tachyon might condense entirely homogeneously in the rest of space-time, leading to a winding-momentum condensate of fundamental strings in the compactified direction. The true vacuum would have at least a $\mathrm{SO}(8,1)$ space-time isometry. The $\mathrm{U}(1)_{L}$ of the circle would be broken by a tachyon condensate, and the $\mathrm{SU}(2)_{R}$ would be unbroken. In the new phase, the energy of space-time is lower than in the old phase, and we might expect a homogeneous negative cosmological constant. A new background of the form $A d S_{9}$ at string scale is consistent with these expectations. There would be space-time symmetry enhancement. The background would be complemented with a tachyon expectation value. Massive string modes and Kaluza-Klein excitations would mix, and would result in a stringy geometry. One can attempt to demonstrate the existence of these backgrounds directly. See e.g. [16, 17].

To get a handle on this phase it might be necessary to have a practical formulation of heterotic string field theory. Since for the heterotic string the most tachyonic direction is easily identified by spectral analysis, the problem seems worthy of attention. String field theory was extremely useful in furthering our understanding of open string tachyon condensation $[18,19]$.

Another avenue of research would be to analyze in this Scherk-Schwarz model how tachyon condensation influences ultraviolet properties of the spectrum through the UV/IR connection in the closed string torus amplitude [20, 21]. ${ }^{3}$

\section{Acknowledgments}

We would like to thank Carlo Angelantonj, Costas Bachas, Mohammad Garousi, Guangyu Guo, Costas Kounnas, Daniel Robbins and Nick Toumbas for interesting discussions and correspondence. Our work was supported in part by the grant ANR-09-BLAN-0157-02. The work of WS was supported by NSF under PHY-0505757 and the University of Texas A\&M. WS would like to thank Ecole Normale Supérieure for kind hospitality during different stages of this project.

Open Access. This article is distributed under the terms of the Creative Commons Attribution Noncommercial License which permits any noncommercial use, distribution, and reproduction in any medium, provided the original author(s) and source are credited.

\footnotetext{
${ }^{3}$ We would like to thank Carlo Angelantonj for a clarifying discussion on this point.
} 


\section{References}

[1] J.J. Atick and E. Witten, The Hagedorn transition and the number of degrees of freedom of string theory, Nucl. Phys. B 310 (1988) 291 [SPIRES].

[2] M.J. Bowick and S.B. Giddings, High temperature strings, Nucl. Phys. B 325 (1989) 631 [SPIRES].

[3] J.L.F. Barbon and E. Rabinovici, Touring the Hagedorn ridge, hep-th/0407236 [SPIRES].

[4] E. Alvarez and M.A.R. Osorio, Superstrings at finite temperature, Phys. Rev. D 36 (1987) 1175 [SPIRES].

[5] C. Kounnas and B. Rostand, Coordinate dependent compactifications and discrete symmetries, Nucl. Phys. B 341 (1990) 641 [SPIRES].

[6] M.A.R. Osorio and M.A. Vazquez-Mozo, Duality in nontrivially compactified heterotic strings, Phys. Rev. D 47 (1993) 3411 [hep-th/9207002] [SPIRES].

[7] M. Dine, E. Gorbatov, I.R. Klebanov and M. Krasnitz, Closed string tachyons and their implications for non- supersymmetric strings, JHEP 07 (2004) 034 [hep-th/0303076] [SPIRES].

[8] I. Antoniadis, J.P. Derendinger and C. Kounnas, Non-perturbative temperature instabilities in $N=4$ strings, Nucl. Phys. B 551 (1999) 41 [hep-th/9902032] [SPIRES].

[9] C. Angelantonj, M. Cardella and N. Irges, An alternative for moduli stabilisation, Phys. Lett. B 641 (2006) 474 [hep-th/0608022] [SPIRES].

[10] C. Angelantonj, C. Kounnas, H. Partouche and N. Toumbas, Resolution of Hagedorn singularity in superstrings with gravito-magnetic fluxes, Nucl. Phys. B 809 (2009) 291 [arXiv:0808.1357] [SPIRES].

[11] I. Antoniadis and C. Kounnas, Superstring phase transition at high temperature, Phys. Lett. B 261 (1991) 369 [SPIRES].

[12] S. Weinberg, Coupling constants and vertex functions in string theories, Phys. Lett. B 156 (1985) 309 [SPIRES].

[13] J. Polchinski, String theory. Vol. 1: An introduction to the bosonic string, Cambridge University Press, Cambridge U.K. (1998).

[14] J.Polchinski, String theory. Vol. 2: Superstring theory and beyond, Cambridge University Press, Cambridge U.K. (1998).

[15] M.R. Garousi, Off-shell extension of S-matrix elements and tachyonic effective actions, JHEP 04 (2003) 027 [hep-th/0303239] [SPIRES].

[16] A.M. Polyakov, Supermagnets and $\sigma$-models, hep-th/0512310 [SPIRES].

[17] J.J. Friess and S.S. Gubser, Non-linear $\sigma$-models with anti-de Sitter target spaces, Nucl. Phys. B 750 (2006) 111 [hep-th/0512355] [SPIRES].

[18] A. Sen, Non-BPS states and branes in string theory, hep-th/9904207 [SPIRES].

[19] A. Sen and B. Zwiebach, Tachyon condensation in string field theory, JHEP 03 (2000) 002 [hep-th/9912249] [SPIRES].

[20] D. Kutasov and N. Seiberg, Number of degrees of freedom, density of states and tachyons in string theory and CFT, Nucl. Phys. B 358 (1991) 600 [SPIRES].

[21] C. Angelantonj, M. Cardella, S. Elitzur and E. Rabinovici, Vacuum stability, string density of states and the Riemann zeta function, JHEP 02 (2011) 024 [arXiv:1012.5091] [SPIRES]. 